\title{
SITIO WEB PARA EL APRENDIZAJE EN LA CARRERA DE INGENIERÍA MECÁNICA
}

\section{WEB SITE FOR LEARNING IN MECHANICS ENGINEERING CAREER}

\author{
Ernesto Rodríguez Alba
}

Departamento de Ingeniería Mecánica de la Universidad de Granma, Cuba.

erodrigueza@udg.co.cu

\author{
Departamento de Ingeniería Mecánica de la Universidad de Granma, Cuba. \\ Yuri Bueno Montana \\ Leudis Villavicencio Lozano
}

Departamento de Informática de la Universidad de Granma, Cuba.

Antonio Mustelier Hechavarría

\author{
Idalberto Macías Socarrás \\ Universidad Estatal Península de Santa Elena (UPSE)
}

\section{RESUMEN}

Una de las problemáticas más acuciantes en todas las universidades del país, actualmente, es lograr estudiantes con una adecuada formación investigativa, para ello se requiere desde el pregrado el diseño e implementación de programas que favorezcan la formación y desarrollo de la misma y permitan instrumentar los cambios necesarios en sus esferas de actuación y campos de acción. Partiendo de esta necesidad el presente trabajo tiene como objetivo la elaboración de un Sitio Web para contribuir a la formación y desarrollo de habilidades investigativas básicas en los estudiantes de Ingeniería Mecánica, a partir del uso de las Tecnologías de la Informática y las Comunicaciones en el entorno docente. Se determinó el contenido fundamental a desarrollar en el mismo a partir de un adecuado diseño, empleando para el mismo la metodología de desarrollo de Software propuesta por Pressman. El análisis realizado mostró que el uso de los Sitios Web puestos a disposición de estudiantes y profesores en los procesos de enseñanza aprendizaje, contribuye a la formación de habilidades básicas como la toma de notas, asentamiento y fichaje de bibliografías y el reconocimiento de problemas en la práctica profesional, herramientas suficientes para hacer de ella una tarea de primer orden en su accionar diario como estudiantes. Basado en el estudio realizado se concluye que el Sitio Web Docente propuesto constituye una aplicación de gran utilidad para el logro de este objetivo, teniendo en cuenta las potencialidades educativas que brinda este recurso informático.

Palabras clave: Tecnologías de la Informática y las Comunicaciones, Sitio Web Docente, Habilidades Investigativas, Recurso Informático, Software

\begin{abstract}
Nowadays one of the most urgent problems for all the universities in the country is to graduate students with an proper research education, for which a design and implementation of programs that foster formation and development in that sense at that level of education and allow to carry out the necessary changes in the students' sphere of performance and fields of action are required. The main goal of this paper is to create a web site in order to contribute to form and develop basic research skills in the Mechanical Engineering students by using information technologies in the educational environment. Main content and a proper design of the site were defined. A suitable software engineering methodology for good practices was followed. The study showed that the adequate use of web sites available to students and teachers in the teaching-learning processes contribute to form and develop basic skills such as note-taking, referring and arranging of bibliographies, and the recognition of problems in the professional practice, powerful tools to make it a first order task in the students' daily performance of the job. The proposed web site turns out to be an useful software for the achievement of this goal, taking into account the educative potentials this informatics resource offers.
\end{abstract}

Keywords: Information Technologies and Communications, Web Site, Investigative skills, computer 
resource, Software.

Recibido: agosto de 2016 Aprobado: septiembre de 2016

\section{Introducción}

Actualmente la actividad científica investigativa del país se caracteriza básicamente por la incorporación de las Tecnologías de la Informática y las Comunicaciones (TIC) al proceso de investigación y una marcada tendencia integradora, donde los resultados son introducidos en la práctica con relativa rapidez por lo que se convierte en una necesidad, el desarrollo y la difusión de una cultura científico investigativa, que contribuya a la educación integral del hombre. Esta situación conlleva a la educación al planteamiento de nuevas exigencias en la formación científica de las futuras generaciones, sobre todo en lo que respecta a la determinación de los problemas que se dan en sus esferas de actuación y campos de acción, así como la búsqueda de alternativas para su solución. Para el logro de esta meta, aún no se cuenta con una respuesta definitiva, respecto a cuáles son las vías que deben seguirse para lograr una adecuada formación investigativa de los estudiantes que una vez egresados les permita, con la capacidad suficiente, lograr un adecuado desempeño profesional.

En este sentido y a pesar de la gran cantidad de recursos empleados todavía no es posible hablar de una correcta formación investigativa de los estudiantes lo que ha quedado evidenciado en las insuficiencias detectadas en los análisis de los resultados de los diferentes trabajos de investigación que realizan a lo largo de la carrera y que tienen su culminación en una tesis o trabajo de diploma las cuales relacionamos a continuación: 1. Insuficiencias en el análisis de situaciones concretas de la práctica relacionadas con la carrera. 2. Incorrecta identificación y formulación de problemas científicos.

3. Insuficiente uso de las TIC en el desarrollo del proceso investigativo.

4. No se explota la intranet del centro para publicar resultados y experiencias de investigación de los alumnos que permitan motivar, estimular y a la vez servir de material de apoyo al resto del alumnado.

En el estudio realizado se analizaron los antecedentes del proceso de formación y desarrollo de las habilidades investigativas, y de los referentes psicológicos, pedagógicos y tecnológicos que sustentan el uso de la Web con fines docentes. Como resultado del cual se obtuvo un sitio Web para el desarrollo de habilidades investigativas que permitirá la elevación de la calidad de la for- mación investigativa.

\section{Materiales y Métodos.}

"Las Tecnologías de la Información y las Comunicación (TIC) son incuestionables y están ahí, forman parte de la cultura tecnológica que nos rodea y con la que debemos convivir. Incluimos en el concepto TIC los medios de comunicación de todo tipo: los medios de comunicación social ("mass media") y los medios de comunicación interpersonales tradicionales con soporte tecnológico como el teléfono, fax..."1

El desarrollo alcanzado por ellas ha convertido al medio informático en parte necesaria del proceso intelectual del hombre. Con cada nuevo descubrimiento en este campo aumenta el volumen de información por lo que se hace necesario poseer la habilidad de saber escoger entre toda esa avalancha de información aquella que realmente necesitamos.

El entorno educacional no ha permanecido ajeno a este desarrollo y un elemento importante en este sentido corresponde al empleo de la computadora como elemento integrante de procesos de enseñanza - aprendizaje que ha ido cobrando cada día una creciente importancia, acentuada precisamente por las potencialidades que nos brindan estos medios al permitir un acceso más expedito a la información proporcionada por Internet y especialmente, de la World Wide Web. En consecuencia, hoy todos los sistemas de educación del mundo, tienen como principal desafío la utilización correcta de estas tecnologías en función de dotar a los estudiantes de las herramientas, conocimientos y habilidades necesarias que se requieren para el desempeño eficiente dentro de lo que actualmente se ha dado en llamar globalización de la información.

\section{Metodología de Desarrollo del sitio Web.}

Según Área Moreira una Web docente es: "un material didáctico distribuido a través de la WWW creado específicamente para el dictado y estudio de una asignatura o materia universitaria. Son Webs de naturaleza didáctica ya que ofrecen un material diseñado y desarrollado específicamente para ser utilizado en un proceso de enseñanza-aprendizaje. En este sentido, pudiéramos indicar que estos sitios Web son materiales curriculares en formato digital que utilizan la WWW como una estrategia de difusión y de acceso al mismo. Suelen ser elaborados por profesores para la enseñanza de su materia y/o asignatura y se corresponderían con el segundo nivel de in- 
tegración de los recursos de Internet en la docencia"2.

Para la elaboración del Sitio Web propuesto tomamos como referencia el modelo de desarrollo de software propuesto por Pressman que se muestra en la figura.

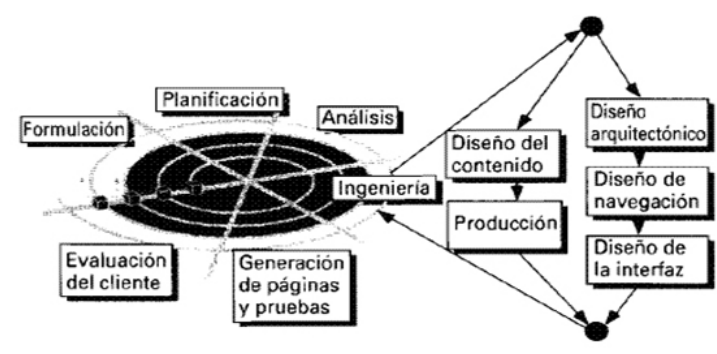

Figura 1. Modelo de desarrollo Web

Comienza con la formulación, actividad en la cual se identifican las metas y los objetivos del proyecto, estableciendo el ámbito para el primer incremento y culmina con la evaluación por parte del cliente donde se revisa y prueba el resultado de cada incremento producido durante el proceso, a la par que se realizan los cambios y ampliaciones necesarios en cada ámbito integrándolos nuevamente mediante el flujo incremental del proceso Tomando en consideración el modelo de proceso Web propuesto por Pressman y las características del proyecto se decidió que durante el diseño del sitio Web, en las primeras fases (formulación, análisis, panificación) se tomarán en cuenta algunas consideraciones antes de comenzar el trabajo con el editor.

En la primera fase se realizó fundamentalmente el trabajo de mesa o sea, frente a una hoja en blanco plantear el proyecto.

Los primeros bocetos en esa primera hoja fueron concebidos con cada uno de los elementos que compondrán el sitio y de lo cual depende el éxito del mismo. En este primer contacto con el proyecto de elaboración del sitio Web se realizaron las siguientes preguntas:

1. ¿Sobre qué trata el sitio? (Breve descripción de los contenidos de la página, su título principal). 2. ¿Finalidad que se persigue al hacerlo? (Fines informativos, entretenimiento, comunicación, formación etc.)

3. ¿Qué se necesita? (Fuentes documentales, Hardware, Software, Equipo humano, Idiomas, soportes)

4. ¿Qué medios existen?

5. Análisis de usuarios (Describir tipo de público (usuarios), objetivo, nivel Informático. etc.)
El objetivo esencial de estas fases fue delimitar la intención global del sitio Web y al ir dando respuesta a cada una de las interrogantes se fue perfilando una idea exacta de la propuesta o del contenido fundamental a tratar en el sitio. Los conceptos y principios que se tratan para el análisis de los requisitos del software también fueron aplicados en la actividad de análisis de ingeniería Web.

\section{Ingeniería}

Según el modelo de Pressman, la actividad de ingeniería incorpora dos tareas paralelas, como se muestra en la Figura.1 "El diseño del contenido y la producción son tareas llevadas a cabo por personas no técnicas del equipo Web. El objetivo de estas tareas es diseñar, producir, y/o adquirir todo el contenido de texto, grafico y video que se vayan a integrar en la Web. Al mismo tiempo, se lleva a cabo un conjunto de tareas de diseño"3

\section{Diseño arquitectónico.}

El sitio propuesto fue montado en una estructura de cliente-servidor utilizando como plataforma soporte servidores Apache. Su arquitectura se basa en una estructura mixta al poseer características de las estructuras lineales y en red. En él, la meta fundamental es hacer corresponder la estructura de la Web con el contenido que se va a presentar. La pantalla principal y las restantes cuentan con un diseño equilibrado en el cual se tuvo en cuenta al tipo de estudiantes al cual está dirigido, y en función de esto se realizó un análisis de los elementos y estructuras de diseño para que sin afectar el contenido se logre un manejo fácil y cómodo de este producto.

\section{Diseño de Navegación.}

La página principal o índice, se diseñó teniendo en cuenta que la misma permitiera lograr el objetivo principal: captar la atención de los visitantes al sitio y revelar el mensaje fundamental del mismo, sus diferentes secciones fueron organizadas fundamentalmente tratando de que constituyeran una guía en la navegación por el sitio y captaran el interés de los visitantes y al mismo tiempo permitiendo a cualquier visitante conocer qué incluye cada sección partiendo de un esquema de navegación lo más funcional posible que permita el desplazamiento libre entre las páginas del sitio de forma tal que resulte fácil la misma para cualquier usuario y en especial para los estudiantes.

A partir de la página de inicio se podrá acceder a las demás que han sido concebidas con marcos que dividirán la pantalla en tres áreas. 
En el marco izquierdo estarán ubicadas las diferentes secciones del sitio con:

- Inicio: Breve resumen de la finalidad del sitio.

- Contenido: En esta sección el estudiante podrá acceder a una pequeña guía de la asignatura, que incluya el programa, la importancia de la asignatura, los temas, apuntes; y video conferencias a través de los cuales irá desarrollando las habilidades correspondientes a cada tema.

- Biblioteca: En ella se podrá acceder a una bibliografía actualizada relacionada con la asignatura, a publicaciones que son resultado de los eventos científicos de estudiantes y profesores, además de monografías publicadas por docentes de varias universidades del país. Estas publicaciones podrán ser utilizadas también como bibliografía y material de consulta.

- Enlaces: A través de ellos podrá acceder directamente a la página oficial de la Universidad dentro de la cual encontrará diferentes opciones y servicios como: correo electrónico para estudiantes, foro, catálogo de artículos, Trabajo Científico Estudiantil (TCE) etc. páginas de interés, bibliotecas virtuales, además, a través de esta también podrá acceder a la de la Facultad de Ciencias Técnicas dentro de la cual encontrará otras opciones, relacionadas con su carrera.

- Ejercicios: Ejercicios interactivos relacionados con cada tema con posibilidades de auto evaluación.

- Glosario: Contiene terminología utilizada en la asignatura y en las investigaciones.

- Interesante: En esta página el estudiante podrá encontrar las convocatorias de los diferentes eventos que se programan en la universidad a los diferentes niveles (fórum, concursos).

El marco derecho mostrará el contenido específico de la página con enlaces para ir a la página anterior, o ir a la siguiente en caso de que el contenido sea muy extenso y volver a la página de la sección.

Las demás páginas se concibieron partiendo del concepto de que ninguna página puede quedar huérfana, es decir todas las páginas deben de tener enlaces. El usuario siempre puede tener una noción de donde se encuentra exactamente dentro del sitio durante la navegación, manteniendo un marco principal, el cual contiene los botones que facilitan la navegación hacia las restantes páginas.

\section{Diseño de la interfaz}

Según Rodríguez Lama, R se denomina interfaz, "...a la zona de comunicación en la que se realiza la interacción entre usuario y programa"4.

En la elaboración de la interfaz gráfica del sitio
Web, se tuvo en cuenta como principio básico la unidad de la imagen gráfica con su significado; el uso de los colores y la iconografía.

Para el logro de este objetivo se elaboró una guía en la que quedaron detallados los elementos esenciales referentes al diseño gráfico y composición que garantizan la coherencia general que incluye:

1. Estructura de los documentos (navegación)

2. Colores identificativos y colores de los fondos.

3. Iconografía.

Todo esto teniendo en cuenta que el objetivo fundamental es que será utilizado por estudiantes universitarios, por tanto, todos los elementos incluidos en su diseño como la iconografía, color, balance de formas y estructuras de diseño deben estar en función del contenido, combinadas de forma tal que logre en los estudiantes una rápida familiarización con el mismo, un manejo fácil y cómodo de este producto.

\section{Producción}

La producción del mismo no se realizó a gran escala puesto que es un software que será utilizado solamente dentro de la universidad y con un objetivo específico; no obstante para su elaboración se realizó un análisis económico de la propuesta en el que se tuvo en cuenta el costo del desarrollo de la misma y se compararon con los beneficios que producirá una vez desarrollado. Estos costos de desarrollo se cuantificaron partiendo del análisis de las inversiones en equipos, horas-hombre necesarias en las fases de análisis, diseño y desarrollo. Este análisis arrojó que los costos de desarrollo del sitio propuesto no son significativos en cuanto a equipos informáticos (computadoras), y no fue necesario remunerar al hombre. Técnicamente el posicionamiento del sitio es viable, dado que en el centro existen los recursos técnicos necesarios para ello, tomando en consideración las características técnicas del sitio Web para su funcionamiento.

\section{Requisitos técnicos}

1. El sitio Web propuesto puede hospedarse en la intranet de la Universidad en una plataforma soporte de servidores Web por la parte del servidor y Preprocesador PHP por la parte del cliente; el usuario puede navegar utilizando (Mozilla Firefox, Internet Explorer), Sistema Operativo: Windows 98 (SE), Millenium, 2000, XP, 2006 ó cualquier aplicación basada en UNIX (Linux), Microprocesador a $300 \mathrm{MHz}$ o superior, controlador de red.

2. La aplicación debe ser totalmente escalable a 
fin de permitir su crecimiento futuro.

3. La aplicación debe probarse rigurosamente antes de ponerse a disposición de la comunidad que usará este sitio.

\section{Indicaciones metodológicas para el uso del Sitio Web.}

El profesor podrá utilizar el sitio Web en función del desarrollo de sus clases, así como en la orientación de tareas docentes y del estudio y/o trabajo independiente, por la variedad y riqueza de ejercicios que posee, también servirá como fuente bibliográfica en sus manos y en la de los estudiantes para garantizar su formación académica, investigativa y laboral.

Se sugiere que por el poco conocimiento sobre el trabajo con redes que poseen los estudiantes en el primer año, los profesores que se desempeñan con ellos, expliquen el funcionamiento del sitio, así como su concepción, si fuera necesario, para garantizar el fin con el cual fue concebido.

Se propone que durante el desarrollo de las actividades y los ejercicios que proponga el profesor, en los diferentes temas, el estudiante trabaje solo; esto posibilitará el desarrollo de su independencia, habilidades informáticas e investigativas y a la vez permitirá al profesor atender las diferencias individuales.

Para la evaluación de los estudiantes, se sugiere la utilización de las actividades, las cuales podrán ser enviadas por el enlace a la dirección de correo del profesor, y la realización de los ejercicios de los diferentes temas en los cuales se tendrá en cuenta:

- Preparación previa de los estudiantes.

- Solución de los ejercicios.

- Organización de las actividades realizadas.

- Resultados de cada actividad.

La evaluación se hará básicamente a través de tareas y ejercicios integradores, que promuevan el debate reflexivo, cuestionador, en el cual se garantice la participación activa del estudiante haciendo énfasis en la autoevaluación y la evaluación, las cuales podrán hacerse frecuentemente en cada clase y mediante las tareas orientadas en los diferentes temas por el profesor.

Para el estudio independiente, el profesor orientará actividades variadas para lo cual podrá apoyarse tanto en los ejercicios de los diferentes temas como en las que propone el programa de la asignatura y otras que él considere, a partir del propio desarrollo del proceso.
Finalmente, el control de los resultados de cada estudiante se realizará a través de la traza que brinda el sistema lo que permite el registro de los resultados del estudiante en las actividades realizadas; además, permite al profesor delimitar con precisión hasta dónde se llegará en cada momento o actividad, definiendo con claridad el contenido de su interés atendiendo al diagnóstico de cada estudiante.

\section{Valoración final}

El Sitio Web desarrollado ha sido puesto a consideración y validado por varios especialistas, para evaluar la efectividad del mismo, tanto en su concepción teórica como de ingeniería de Software, reconociendo que como proyecto está listo, el mismo que puede generar beneficios a la práctica educativa como:

1. Una mayor cantidad, calidad y rapidez de acceso a la información, permitiendo a los usuarios poseer una mayor cantidad de información relevante y actualizada.

2. Cambios en los modos de trabajo y de tratamiento de los contenidos.

3. Aumento de la calidad en el desarrollo de la docencia en la carrera.

4. Posibilidad del desarrollo del proceso de enseñanza aprendizaje utilizando métodos predominantemente activos.

5. Perfeccionamiento de la concepción didáctico-metodológica del proceso de formación investigativa.

6. Mayor relación entre los componentes académico, investigativo y laboral y dentro de este el uso de las TIC como herramienta de trabajo.

\section{Conclusiones}

En el caso específico del Sitio Web desarrollado y propuesto en este trabajo podemos concluir:

1. La metodología utilizada permitió fundamentar el tipo de Software a diseñar, justificar la tecnología que lo soporta y su portabilidad para varias plataformas, el alcance en cuanto a números de usuarios, recursos invertidos y funcionalidad confirmando que el Sitio Web propuesto puede ser utilizado en el proceso docente educativo de la carrera de Ingeniería Mecánica, como un medio de enseñanza interactivo para contribuir a la formación y desarrollo de las habilidades investigativas básicas en los estudiantes.

2. El Sitio Web propuesto para ser utilizado en el proceso de enseñanza-aprendizaje constituye un medio de enseñanza de gran utilidad para contribuir a la formación y desarrollo de las habilidades investigativas en el primer año de la carrera. 


\section{Referencias bibliográficas}

1. Las TIC y sus aportaciones a la sociedad [homepage en Internet] Barcelona, España; c2008 [actualizada 25 mayo 2016; consultado 25 mayo 2016]. Disponible en:https://www.researchgate. net/profile/Pere_Graells/publication/267419766 LAS_TIC_Y_SUS_APORTACIONES_A_LA_SOCIEDAD/links/55b7702408ae9289a08be5ff.pdf 2. Internet en la docencia universitaria. Web docentes y aulas virtuales. [homepage en Internet] Universidad de la Laguna, Santa Cruz de Tenerife, España; c2005 [actualizada 25 mayo 2016; consultado 25 mayo 2016]. Disponible en: https:// www.um.es/c/document_library/get_file?uuid=eaca8858-516f-4718-ab1b-76a4f057bc65\&groupld $=316845$

3. S Pressman, R. Ingeniería de Software. Un enfoque práctico (4ta Edición ed.). México: McGraw Hill; 1998.

4. Rodríguez Lama, R. y. o. Introducción a la Informática Educativa. La Habana Cuba: Pueblo y Educación; 2000

\section{Bibliografía}

1. Chirino Ramos, M. V. La investigación en el desempeño profesional pedagógico, Profesionalidad y Práctica Pedagógica. La Habana: Pueblo y Educación; 2004

2. Fariñas León, G. Desafíos de la investigación educativa: presente y futuro. Metodología de la investigación educacional. La Habana Pueblo y educación; 2005.

3. Echeverría, J. Apropiación social de las tecnologías de la información y la comunicación. Revista CTS. 2008; 4: 171-182.

4. González Castro, V. Teoría y práctica de los medios de enseñanza. La Habana: Pueblo y Educación; 1986.

5. González Castro, V. (1979). Los Medios de enseñanza. Ciudad de la Habana: Pueblo y Educación.

6. Ministerio de Informática y las Comunicaciones. Estrategia para la Informatización de la Sociedad Cubana hasta el año 2000. La Habana: MIC; 2001.

7. Ministerio de Educación. Las tecnologías de la información y la comunicación en la formación inicial y permanente. La Habana: MINED; 2007

8. S Pressman, R. (1998). Ingeniería de Software. Un enfoque práctico (4ta Edición ed.). México: McGraw Hill. 